\title{
Outcomes of gastrointestinal bleeding in patients with left ventricular assist devices: a tertiary care experience
}

\section{다 (우우}

\begin{abstract}
Authors McNitt $^{2}$, Truptesh Kothari ${ }^{1}$, Vivek Kaul ${ }^{1}$, Shivangi Kothari ${ }^{1}$

Institutions

1 Center for Advanced Therapeutic Endoscopy, Division of Gastroenterology \& Hepatology, University of Rochester Medical Center, Rochester New York, United States

2 Division of Cardiology, University of Rochester Medical Center, Rochester New York, United States
\end{abstract}

Caren Taylor ${ }^{1}$, Krystle Bittner ${ }^{1}$, Nicholas Bartell', Jose Aranez ${ }^{1}$, Jeffrey D Alexis ${ }^{2}$, Beth Carlson ${ }^{2}$, Leway Chen ${ }^{2}$, Scott

submitted 18.4.2019

accepted after revision $\quad 28.10 .2019$

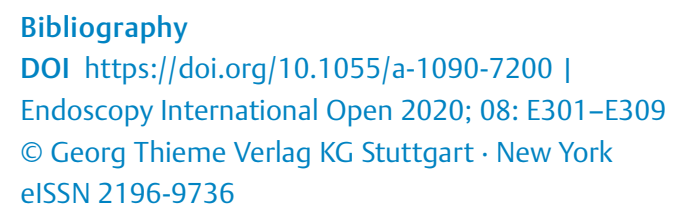

Corresponding author

Dr. Shivangi Kothari, University of Rochester -

GI \& Hepatology, 601 Elmwood Ave Box 646, Rochester,

NY 14627-0001

Fax: +1-585-275-8118

Shivangi_kothari@urmc.rochester.edu

\section{ABSTRACT}

Background and study aims Left ventricular assist device (LVAD) placement is a therapeutic modality for patients with end-stage heart failure. Gastrointestinal bleeding is a common complication following LVADimplantation. The aim of this study was to report our experience in management and outcomes of gastrointestinal bleeding in a large cohort of patients with LVADs.

Patients and methods We performed a retrospective review of all patients who underwent LVAD implantation at the University of Rochester Medical Center from January 2008 to June 2017. Data were collected on patient characteristics, clinical aspects of gastrointestinal bleeding events, and procedural interventions. A Cox proportional hazard model was utilized to identify potential risk factors for a gastrointestinal bleeding event.

Results During the study period, 345 patients underwent LVAD implantation. Of these, 125 patients (36.2\%) experienced 297 gastrointestinal bleeding events resulting in 533 endoscopic procedures. The diagnostic yield of endoscopy in determining a bleeding source was $49.5 \%$. If required, therapeutic interventions were successful in achieving homeostasis in $96.2 \%$ of procedures. Our 30-day overall post-procedure adverse event (AE) rate was $6.6 \%$. Procedure-related (bleeding, infection, and perforation) AES were very minimal (2.8\%). A Cox proportional hazard model indicated that older age at implant, female sex, AfricanAmerican race, diabetes mellitus, and pulmonary hypertension were statistically significant predictors of a gastrointestinal bleeding event following LVADimplantation.

Conclusions LVAD patients have a high risk of gastrointestinal bleeding. Endoscopy was able to safely locate a bleeding lesion in approximately half of our patients and was successful in treating bleeding lesions in a majority of the cases.

\section{Introduction}

Heart failure (HF) has an estimated prevalence of $2.2 \%$ in the United States, with an expected increase of $3 \%$ to $4 \%$ in the next 20 years [1,2]. Left ventricular assist device (LVAD) implantation is the standard of care in end-stage HF refractory to medical management either as a bridge to transplant or as destination therapy. Currently, nearly $50 \%$ of LVADs are being implanted as destination therapy [3]. One of the most common adverse events (AEs) in the post-LVADimplantation period is gastrointestinal bleeding, with a reported prevalence of $14.8 \%$ to $23.0 \%$ [4-9].

Pathogenesis of a gastrointestinal bleeding event in the LVAD patient population is not completely understood; however, it is often reported that gastrointestinal bleeding events occur due to development of a gastrointestinal angiodysplasia (GIAD) [9-11]. The pathophysiological mechanism underlying gastrointestinal bleeding occurrence in patients with LVAD may be similar to Heyde's Syndrome, in that patients with aortic stenosis are at a higher risk of gastrointestinal bleeding [12]. In patients with an LVAD, the normal pulse pressure is dimin- 
ished and is comparable to severe aortic stenosis. A loss of pulse pressure is believed to create alterations in hemodynamics that trigger the development of angiodysplasias [12,13]. In addition, reduction in pulse pressure creates shear forces that can contribute to occurrence of an acquired von Willebrand disease (vWD) [14]. In vivo reductions in von Willebrand factor (vWF) have been confirmed in LVAD patients, with normalization of levels post-heart transplant [15]. Anacquired vWD produces a coagulopathy, which increases overall bleeding risk that is compounded by the requirement for prophylactic anticoagulation for all LVAD patients. Cohort studies examining preimplantation risk factors for development of gastrointestinal bleeding events in patients with LVADs have produced mixed results. Several studies have reported that increased age at time of LVAD placement may increase risk of a subsequent gastrointestinal bleeding [6,16-19]. Other studies have indicated that a history of gastrointestinal bleeding, use of the LVADas destination therapy, or right heart dysfunction are independent risk factors for gastrointestinal bleeding in LVAD patients [2023].

Most suggested management approaches advocate for endoscopic evaluation and intervention, with a reported diagnostic yield ranging from $30 \%$ to $71 \%$ for gastrointestinal bleeding in the LVADpopulation [6,18,24-27]. There is a school of thought that endoscopic management may have limited utility in the LVAD population, as a majority present with an occult gastrointestinal bleeding, making diagnosis more difficult with conventional endoscopic techniques [28]. This limitation may be overcome with early use of device-assisted enteroscopy (DAE) to directly visualize more of the small bowel. A recent systematic review reported that performing DAE early in the course of assessment for suspected gastrointestinal bleeding in this population is associated with decreased transfusion requirements, decreased time to endoscopic intervention, and a high diagnostic yield [29]. In addition, use of video capsule endoscopy (VCE) may further increase diagnostic yield as VCE allows the endoscopist to narrow down the location of a gastrointestinal bleeding source prior to performing DAE. Although the literature describing the utility of endoscopy in assessment of a gastrointestinal bleeding in the LVAD patient is increasing, there is still considerable disagreement on its utility versus conventional management.

The aim of this study is to report our experience in endoscopic management and outcomes of gastrointestinal bleeding in the LVAD population at an academic tertiary care setting. To date, our cohort represents the largest single-center gastrointestinal bleeding data in the LVAD population reported in the literature. Specific areas of interest were to identify potential risk factors for gastrointestinal bleeding and describe outcomes and safety of endoscopic management of gastrointestinal bleeding in LVAD patients.

\section{Patients and methods}

\section{Study population \& data collection}

We retrospectively reviewed electronic medical records of all patients who underwent implantation of an LVAD at the University of Rochester Medical Center (Rochester, NY) between January 2008 and June 2017 to identify those that were admitted with gastrointestinal bleeding (gastrointestinal bleeding). Each gastrointestinal bleeding event was considered independent, and thus analyzed separately. Data were obtained on clinical presentation at time of gastrointestinal bleeding, length of each hospitalization, relevant laboratory studies, and requirements for transfusion per gastrointestinal bleeding event. In addition, data were collected on all endoscopic and radiologic procedures performed during each hospitalization, location of gastrointestinal bleeding (if source identified), endoscopic interventions (if applicable), and post-procedural (30-day) AE rates. An identifiable source of bleeding was defined as: a lesion seen during an endoscopic exam that had evidence of active or recent bleeding that was documented by the endoscopist to be significant enough to cause the patient's clinical presentation. This study protocol was reviewed and approved by the University of Rochester Medical Center institutional review board. All patients who underwent an endoscopic procedure had proper informed consent taken in accordance with institutional policies.

\section{Statistical methods}

Baseline clinical characteristics were compared between patients with or without gastrointestinal bleeding events during follow-up. Continuous measures were expressed as mean \pm SD and range while categorical data were summarized as frequencies and percentages. Statistical comparisons were performed using the Wilcoxon rank-sum test for continuous variables and chi-square test for dichotomous variables, as appropriate. Diagnostic yield was calculated as the number of endoscopic procedures that revealed a bleeding source/total number of endoscopic procedures (note: procedures performed after a source of bleeding was located were not included in this calculation).

Success rate of endoscopic intervention(s) was defined as the number of procedures wherein hemostasis was achieved/ total number of procedures with endoscopic intervention (note: there were several procedures with more than one endoscopic intervention, for the purpose of this calculation, each procedure with an intervention was only counted once toward the denominator). Thirty-day AE event rate was defined as any $\mathrm{AE}$ occurring within 30 days of the completion of the procedure. Each procedure was considered independently. For the vast majority of subjects with complete follow-up data $(n=$ 330), survival analysis techniques were utilized. The cumulative probability of gastrointestinal bleeding was displayed using the Kaplan-Meier method and statistical significance was determined to compare different groups with the log-rank test. Multivariate Cox proportional hazards regression models were used to model the time-to-event endpoints of index gastrointestinal bleeding and mortality. Covariates associated with predicting risk of these endpoints were determined employing the "best 
subsets" regression methodology. Specifically, the best subsets method of variable reduction examines the best models containing one, two, or three variables, and so on, and makes comparisons based on the global score chi-square statistic. In addition, variables needed to be significant at $P<0.10$ for inclusion in the model. For the mortality endpoint, gastrointestinal bleeding was modeled as a time-dependent covariate in the proportional hazards regression model. Analyses were performed using Microsoft Excel, SPSS Version 24 (IBM) and SAS 9.4 .

\section{Results}

A total of 345 patients underwent LVADimplantation during the study period, with 125 (36.2\%) having at least one gastrointestinal bleeding event. Each gastrointestinal bleeding event was recorded independently $(n=297)$. Patients experienced a median of two bleeds with a median time-to-index gastrointestinal bleeding of 0.54 years (range $=0-6.24$ years). Patient characteristics are described in $>$ Table 1. Statistical analyses revealed that those with a gastrointestinal bleeding were more likely to be older (60.0 vs. $54.4 ; P<0.001)$, of African-American race $(21.0 \%$ vs. $10.7 \%$; $P=0.05)$, diagnosed with Type 2 diabetes mellitus (DM-II) (36.8\% vs. $26.8 \% ; P=0.05$ ), pulmonary hypertension ( $12.0 \%$ vs. $5.0 \% ; P=0.02$ ), or chronic kidney disease $(32.8 \%$ vs. $19.1 \% ; P<0.001)$; and to have ischemic cardiomyopathy as an indication for LVADimplant $(46.3 \%$ vs. $31.5 \% ; P=0.02$ ).

As demonstrated in the KM graphs (> Fig.1, > Fig.2, - Fig. 3), younger patients $(\leq 60)$ had cumulative probability of $24 \%$ at 3 years while older patients had a probability of $56 \%$. Similarly, patients who were not diabetic at baseline had a 3-year cumulative probability of gastrointestinal bleeding of $32 \%$ while those who were diabetic had rate of $53 \%$.

Results of the multivariate Cox proportional hazards models for index gastrointestinal bleeding and mortality are shown in - Table 2 and $>$ Table 3. Predictors associated with risk of gastrointestinal bleeding were age at implant, sex, African-American race, diabetes, pulmonary hypertension, and history of acute MI. Risk of a gastrointestinal bleeding event increased by $6 \%$ for each year older at the time of implant. Male patients exhibited a $37 \%$ lower risk than females. Patients of AfricanAmerican race had more than double (2.75 times) the risk of developing a gastrointestinal bleeding. Comorbidities of DM-II and pulmonary hypertension each independently elevated risk of gastrointestinal bleeding by approximate two-fold (HRs 1.8 and 2.2, respectively) while history of acute MI halved the risk. For the mortality endpoint, even after adjustment for age at implant, diabetes and NYHA class, time-dependent gastrointestinal bleeding significantly increased risk of mortality (HR 2.36, 1.58-3.53). When adjusting for baseline Hemoglobin, these results were similar (over $20 \%$ were missing this biomarker and so were included in primary analysis).

Characteristics of each independent gastrointestinal bleeding event are portrayed in $>$ Table 4 . In patients hospitalized for their gastrointestinal bleeding event, median length of stay (LOS) was 8 days (range $0-173$ days). The majority of patients
(59.2\%) were readmitted for a subsequent gastrointestinal bleeding event. Median time to readmission (following prior hemostasis, if the patient had multiple readmissions for gastrointestinal bleeding events) was 118 days. Upon presentation for gastrointestinal bleeding evaluation, median hemoglobin concentration was $6.9 \mathrm{~g} / \mathrm{dL}$ (Range 4.0-15.0), and a median INR of 2.0 (Range 1.0-8.6). Patients were transfused with a median of four units of packed red blood cells per admission for gastrointestinal bleeding, using standard transfusion thresholds of hemoglobin less than $7.0 \mathrm{~g} / \mathrm{dL}$.

Procedural characteristics are described in > Table 5. To evaluate LVAD patients with a suspected gastrointestinal bleeding, 533 endoscopic procedures were performed. At time of their gastrointestinal bleeding event, the majority of patients were on an antithrombotic regimen (97\%), with the most common being a combination of warfarin plus aspirin (73\%). The most common presentations of gastrointestinal bleeding were melena ( $n=142 ; 47.8 \%)$ and symptomatic anemia, without overt signs of gastrointestinal bleeding ( $n=77 ; 25.9 \%$ ). If a source of bleeding was determined, the location was most often in the stomach $(n=113 ; 39.4 \%)$ or small bowel $(n=83$; $28.9 \%$ ). GIADs were the most frequent endoscopic finding ( $n=$ $121 ; 42.4 \%$ ). Our overall diagnostic yield for endoscopic evaluation of gastrointestinal bleeding was $49.5 \%$. A total of 226 interventions were performed, with the most common being argon plasma coagulation ( $n=77 ; 34.1 \%$ ) or endoclip placement $(n=67,29.6 \%)$. The success rate in achieving hemostasis by performing endoscopic interventions was $96.2 \%$. Procedurerelated AEs were very minimal (2.8\%) in our cohort. Thirteen post-procedure bleeds were noted; however, it was difficult to delineate if these were a continuation of their index bleeding event. Perforation ( $n=1$ ) occurred in the rectosigmoid, during colonoscopy and was managed successfully with endoscopic Ovesco clip closure. One case of acute phlebitis was seen following peripheral intravenous placement and was managed conservatively with a 10-day course of antibiotics. Thirty-day post-procedure AEs included LVAD pump thrombosis (0.38\%), cerebrovascular accident (CVA; $0.75 \%$ ), and death (2.6\%). No reported deaths were associated with endoscopic procedures or interventions.

\section{Discussion}

Gastrointestinal bleeding is the most common, long-term AE post-LVAD placement and can lead to significant morbidity and need for repeated endoscopic procedures. To our knowledge, this cohort represents the largest single-center report on endoscopic management of gastrointestinal bleeding events in the LVAD population. Our study confirms that gastrointestinal bleeding is a very common AE following LVADimplantation (36.2\%; $n=125 / 345)$. Interestingly, our cohort had a higher rate of bleeding in LVADpatients as compared to national averages (36.2\% vs. $14.8-23.0 \%$ in prior literature, potentially related to the significantly longer time patients spend in our region waiting for heart transplantation, coupled with the increasing number of LVAD devices being implanted as destination therapy [29]. Although moderate in severity, these gastro- 
- Table 1 Patient Characteristics in LVAD patients with and without gastrointestinal bleeding events.

\begin{tabular}{|c|c|c|c|c|}
\hline & $\begin{array}{l}\text { Total population } \\
(n=345)\end{array}$ & $\begin{array}{l}\text { Non-gastrointestinal } \\
\text { bleeding }(n=220)\end{array}$ & $\begin{array}{l}\text { Gastrointestinal } \\
\text { bleeding }(n=125)\end{array}$ & $P$ value \\
\hline Age at LVAD implant (mean, SD) & $56.4(12.0)$ & $54.4(12.6)$ & $60.0(9.9)$ & $<0.001$ \\
\hline - Range, years & $18-86$ & $18-81$ & $22-86$ & \\
\hline \multicolumn{5}{|l|}{ Sex } \\
\hline - Male & $277(80.3 \%)$ & $180(81.8 \%)$ & $97(77.6 \%)$ & 0.34 \\
\hline - Female & $68(19.7 \%)$ & $40(18.2 \%)$ & $28(22.4 \%)$ & \\
\hline \multicolumn{5}{|l|}{ Race $(n=339)$} \\
\hline - White & $288(84.9 \%)$ & $190(88.4 \%)$ & $98(79.0 \%)$ & 0.05 \\
\hline - African-American & $49(14.5 \%)$ & $23(10.7 \%)$ & $26(21.0 \%)$ & \\
\hline - Other & $2(0.59 \%)$ & $2(0.93 \%)$ & $0(0 \%)$ & \\
\hline BMI at LVAD implant (mean, SD) & $29.5(6.0)$ & $29.5(5.4)$ & $29.4(6.9)$ & 0.94 \\
\hline - Range, BMI (kg/m²) & $14.2-72.7$ & $16.9-48.0$ & $14.2-72.7$ & \\
\hline \multicolumn{5}{|l|}{ Comorbidities at LVAD implantation } \\
\hline . $\mathrm{CHF}$ & $303(87.8 \%)$ & $190(86.3 \%)$ & $113(90.4 \%)$ & 0.27 \\
\hline - $\operatorname{HTN}(n=344)$ & $170(49.4 \%)$ & $100(45.7 \%)$ & $70(56.0 \%)$ & 0.07 \\
\hline - CAD & $168(48.7 \%)$ & $101(45.9 \%)$ & $67(53.6 \%)$ & 0.17 \\
\hline - Hyperlipidemia & $165(47.8 \%)$ & $100(45.4 \%)$ & $65(52.0 \%)$ & 0.24 \\
\hline - DM-II & $105(30.4 \%)$ & $59(26.8 \%)$ & $46(36.8 \%)$ & 0.05 \\
\hline . CKD & $83(33.9 \%)$ & $42(19.1 \%)$ & $41(32.8 \%)$ & $<0.001$ \\
\hline - History of MI & $70(20.3 \%)$ & $40(18.2 \%)$ & $30(24.0 \%)$ & 0.20 \\
\hline - COPD & $51(14.8 \%)$ & $29(13.2 \%)$ & $22(17.6 \%)$ & 0.27 \\
\hline - OSA & $53(15.4 \%)$ & $32(14.5 \%)$ & $21(16.8 \%)$ & 0.58 \\
\hline . LVH & $48(13.9 \%)$ & $32(14.5 \%)$ & $16(12.8 \%)$ & 0.65 \\
\hline - Pulmonary HTN & $26(7.5 \%)$ & $11(5.0 \%)$ & $15(12.0 \%)$ & 0.02 \\
\hline - CVA & $22(6.4 \%)$ & $14(6.4 \%)$ & $8(6.4 \%)$ & 0.99 \\
\hline - History of thrombus/thromboembolic event & $23(6.7 \%)$ & $17(7.8 \%)$ & $6(4.8 \%)$ & 0.30 \\
\hline \multicolumn{5}{|l|}{ Indication for LVAD Implant $(n=340)$} \\
\hline - Ischemic cardiomyopathy & $125(36.8 \%)$ & $69(31.5 \%)$ & $56(46.3 \%)$ & 0.02 \\
\hline - Non-ischemic cardiomyopathy & $127(37.4 \%)$ & $83(37.9 \%)$ & $44(36.4 \%)$ & \\
\hline - Acute MI & $50(14.7 \%)$ & $39(17.8 \%)$ & $11(9.1 \%)$ & \\
\hline - Other & $38(11.2 \%)$ & $28(12.8 \%)$ & $10(8.3 \%)$ & \\
\hline \multicolumn{5}{|l|}{ LVADType $(n=344)$} \\
\hline - Heartmate-II & $306(89.0 \%)$ & $192(87.2 \%)$ & $114(91.9 \%)$ & 0.24 \\
\hline - Heartmate-III & $13(3.8 \%)$ & $9(4.1 \%)$ & $4(3.2 \%)$ & \\
\hline - Heartware & $6(1.7 \%)$ & $3(1.4 \%)$ & $3(2.4 \%)$ & \\
\hline - Other & $19(5.5 \%)$ & $16(7.3 \%)$ & $3(2.4 \%)$ & \\
\hline LVAD for bridge to ttransplant $(n=307)$ & $179(58.3 \%)$ & $116(59.5 \%)$ & $63(56.3 \%)$ & 0.58 \\
\hline
\end{tabular}

BMI, body mass index; CHF, congestive heart failure; HTN, hypertension; CAD, coronary artery disease; DM-II, diabetes mellitus, Type 2; CKD, chronic kidney disease; MI, myocardial infarction; COPD, chronic obstructive pulmonary disease; OSA, obstructive sleep apnea; LVH, left ventricular hypertrophy; CVA, cerebrovascular accident.

Statistical analysis demonstrated significant association between older age $(P<0.001)$, African American race $(P=0.05)$, Type 2 diabetes $(P=0.05)$, chronic kidney disease $(P<0.001)$, pulmonary hypertension $(P=0.02)$, and ischemic cardiomyopathy as the indication for LVAD implant $(P=0.02)$ and development of gastrointestinal bleeding in the post-LVAD implantation course. 


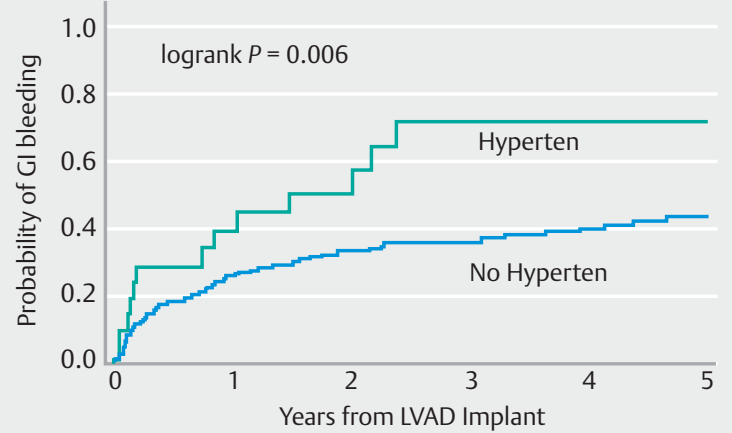

Patients at risk

No Hyperten $308179(0.26) 132(0.33) 87(0.35) 59(0.40) 39(0.43)$

$\begin{array}{lllllll}\text { Hyperten } & 22 & 11(0.39) & 7(0.50) & 2(0.72) & 2(0.72) & 2(0.72)\end{array}$

- Fig. 1 Cumulative probability of gastrointestinal bleeding by hypertension group,

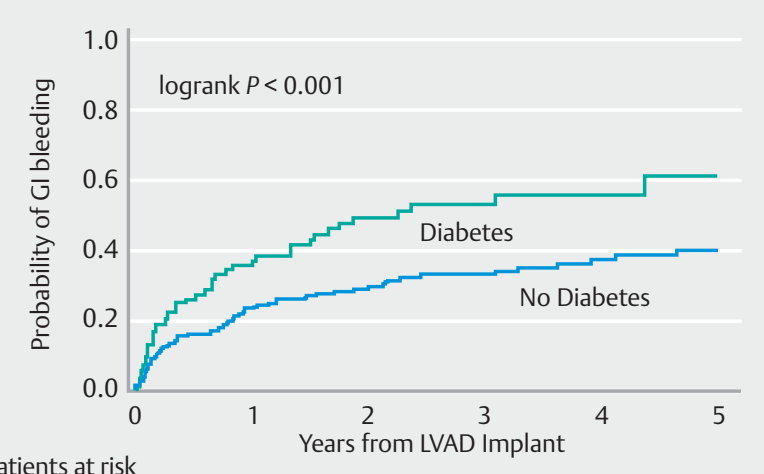

Patients at risk

No Diabetes $237146(0.23) 108(0.28) 71(0.32) 49(0.37) 36(0.40)$

Diabetes $93 \quad 44(0.37) \quad 31(0.49) \quad 18(0.53) \quad 12(0.56) \quad 5(0.61)$

Fig. 2 Cumulative probability of gastrointestinal bleeding by diabetes group,

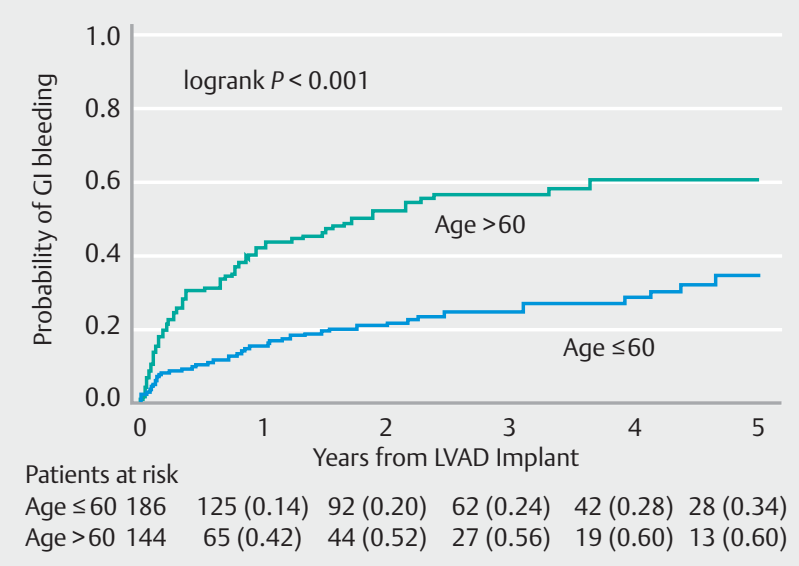

- Fig. 3 Cumulative probability of gastrointestinal bleeding by age groups.

intestinal bleeding events may increase risk of morbidity due to lengthy hospitalizations and the high propensity to develop recurrent gastrointestinal bleedings, often resulting in need for more endoscopic interventions. Endoscopy was demonstrated to be a safe and effective diagnostic and therapeutic modality to manage bleeding lesions in the LVAD population in our study. Nearly half the endoscopic procedures (49.5\%) identified a source of bleeding, with an interventional success rate (to achieve hemostasis) of $96.2 \%$. No reported deaths were associated with endoscopic procedures or interventions in our study. A recent retrospective study reviewed a cohort of 87 patients with LVADs implanted at a tertiary care center with a total of 164 gastrointestinal bleeding events [28]. The reported diagnostic yield of endoscopy was significantly lower (30\%) as compared to the current study. Given these findings, the authors recommended against routine endoscopic evaluation for occult gastrointestinal bleeding events, unless hemodynamic instability is present or significant transfusions are required; however, with a higher diagnostic yield during the episode of

- Table 2 Cox proportional hazards multivariate models for the endpoint of first gastrointestinal bleed.

\begin{tabular}{|l|l|l|l|l|}
\hline Parameter & P value & Hazard & $\mathbf{9 5 \%} \mathbf{C l}$ & UCL \\
\hline & & ratio & LCL & 1.08 \\
\hline Age at implant & $<.0001$ & 1.06 & 1.04 & 1.01 \\
\hline Male & 0.054 & 0.63 & 0.40 & 4.49 \\
\hline African-American race & $<.0001$ & 2.75 & 1.68 & 2.61 \\
\hline DMII baseline & 0.004 & 1.77 & 1.20 & 3.82 \\
\hline Pulmonary HTN baseline & 0.012 & 2.12 & 1.18 & 1.05 \\
\hline Acute MI & 0.072 & 0.56 & 0.30 & 0.70 \\
\hline Hemoglobin B & 0.030 & 0.87 & 0.76 \\
\hline Cl, confidence interval; LCL, confidence limit; UCL, upper confidence limit; DM-II, diabetes mellitus, Type 2; HTN, hypertension; MI, myocardial infarction
\end{tabular}


Table 3 Cox proportional hazards multivariate models for the endpoint of all-cause mortality.

\begin{tabular}{|l|l|l|l|}
\hline Parameter & P value & Hazard & L5\% CI \\
\hline & & ratio & UCL \\
\hline Age at implant & 0.009 & 1.03 & 1.01 \\
\hline DMII baseline & 0.042 & 1.47 & 1.01 \\
\hline NYHA Class & 0.007 & 1.89 & 1.19 \\
\hline Td gastrointestinal bleed & $<.0001$ & 2.36 & 1.58 \\
\hline Td gastrointestinal bleed & 0.003 & 2.10 & 1.30 \\
\hline Hemoglobin b & 0.022 & 0.87 & 3.53 \\
\hline $\begin{array}{l}\text { Cl, confidence interval; LCL, lower confidence limit; UCL, Upper confidence limit ; DM-Il, diabetes mellitus, type 2; NYHA, New York Heart Association; Td, time } \\
\text { dependent }\end{array}$ & & 3.41 \\
\hline
\end{tabular}

- Table 4 Gastrointestinal bleeding event characteristics.

\begin{tabular}{|c|c|}
\hline Event characteristics & Median \\
\hline $\begin{array}{l}\text { Time to first gastrointestinal bleeding event post- } \\
\text { LVAD, years } \\
\text { Range, years }\end{array}$ & $\begin{array}{l}0.5 \\
0-6.24\end{array}$ \\
\hline $\begin{array}{l}\text { LOS, days } \\
\text { Range, days }\end{array}$ & $\begin{array}{l}8 \\
0-173\end{array}$ \\
\hline $\begin{array}{l}\text { Time to readmission, days }{ }^{1} \\
\text { Range, days }\end{array}$ & $\begin{array}{l}118 \\
1-1845\end{array}$ \\
\hline $\begin{array}{l}\text { Number of readmissions } \\
\text { Range }\end{array}$ & $\begin{array}{l}2 \\
1-14\end{array}$ \\
\hline $\begin{array}{l}\mathrm{HCT}, \% \text { at time of gastrointestinal bleeding } \\
\text { Event range }\end{array}$ & $\begin{array}{l}22.0 \\
14.0-45.0\end{array}$ \\
\hline $\begin{array}{l}\mathrm{HgB}, \mathrm{g} / \mathrm{dL} \text { at time of gastrointestinal bleeding } \\
\text { Event range }\end{array}$ & $\begin{array}{l}6.9 \\
4.0-15.0\end{array}$ \\
\hline $\begin{array}{l}\text { INR at time of gastrointestinal bleeding event } \\
\text { Range }\end{array}$ & $\begin{array}{l}2 \\
1.0-8.6\end{array}$ \\
\hline $\begin{array}{l}\text { Platelets at time of gastrointestinal bleeding Event } \\
\text { range }\end{array}$ & $\begin{array}{l}213 \\
27-571\end{array}$ \\
\hline $\begin{array}{l}\text { PRBC units given } \\
\text { Range }\end{array}$ & $\begin{array}{l}4 \\
1-39\end{array}$ \\
\hline $\begin{array}{l}\text { FFP units given } \\
\text { Range }\end{array}$ & $\begin{array}{l}2 \\
1-8\end{array}$ \\
\hline $\begin{array}{l}\text { Platelets given } \\
\text { Range }\end{array}$ & $\begin{array}{l}1.5 \\
1-5\end{array}$ \\
\hline \multicolumn{2}{|c|}{$\begin{array}{l}\text { LOS, length of stay; } \mathrm{HCT} \text {, hematocrit, } \mathrm{HgB} \text {, hemoglobin; INR, international } \\
\text { normalized ratio; PRBC, packed red blood cells; FFP, fresh frozen plasma. } \\
\text { During the study period, } 125 \text { patients had gastrointestinal bleeding events. } \\
\text { There were } 297 \text { independent bleeding events with a median of two events } \\
\text { per patient. Median time from LVAD implant to first gastrointestinal bleed- } \\
\text { ing event was } 0.5 \text { years. Median length of stay for gastrointestinal bleeding- } \\
\text { associated hospitalizations was } 8 \text { days. } 59.2 \% \text { of patients were readmitted } \\
\text { for a subsequent gastrointestinal bleeding event, at a median of } 118 \text { days } \\
\text { (following prior hemostasis, if the patient had multiple readmissions). } \\
1 \mathrm{n}=74 / 125 \text { patients had at least one subsequent readmission (gastrointes- } \\
\text { tinal bleeding-related). }\end{array}$} \\
\hline
\end{tabular}

gastrointestinal bleeding, there is clear value of endoscopy in the routine management LVAD patients with a gastrointestinal bleeding event.

Pathophysiology of bleeding in LVAD patients is multifactorial with acquired von Willebrand factor deficiency, hemodynamic flow alterations, and coagulopathy with need for ongoing anticoagulation to prevent pump dysfunction/thrombosis. The majority of the bleeding in these patients is due to GIAD; however, several studies have also reported peptic ulcer disease as a very common source of bleeding in the LVAD patients due to nonsteroidal anti-inflammatory drug (NSAID)-induced damage to the gastrointestinal tract mucosa coupled with platelet inhibitor use and anticoagulation [30].

Endoscopy remains the mainstay in the evaluation of gastrointestinal bleeding events in these patients, with our study indicating upper endoscopy to have the highest diagnostic yield. Push enteroscopy can be considered as an early intervention in recurrent gastrointestinal bleeding events, specifically in patients who present with melena, iron deficiency anemia or occult gastrointestinal bleeding (as the location of the bleeding lesion is most often in the small bowel. This approach is supported in the literature, as it has been reported that performing an enteroscopy early in the course of a gastrointestinal bleeding event may reduce transfusion requirements and increase endoscopic diagnostic yield [31]. For hemodynamically stable patients and those with a negative upper and lower endoscopic evaluation with persistent gastrointestinal bleeding, a VCE with or without computed tomography enterography or a tagged red blood cell scan should be performed. A device-assisted enteroscopy (DAE) would follow, as information gained from VCE and/or radiologic exams would provide the endoscopist with an appropriate target for the procedure. Multiple algorithms have been proposed in the literature [28,32,33] for evaluation of gastrointestinal bleeding in LVAD patients; however, there is no standardized guideline for evaluation of these patients and data regarding screening high-risk patients for bleeding prior to LVAD placement are scarce. A recent study reviewed 64 gastrointestinal bleeding events in LVAD patients to evaluate risk of mortality after the index gastrointestinal bleeding event [34]. Their findings suggest increased mortality after 
- Table 5 Endoscopic procedural characteristics.

\begin{tabular}{|c|c|}
\hline $\begin{array}{l}\text { Procedural characteristics } \\
\text { ( } n=297 \text { gastrointestinal bleeding) }\end{array}$ & $\mathrm{n}(\%)$ \\
\hline$\%$ of procedures performed on inpatients & $257(86.5 \%)$ \\
\hline \multicolumn{2}{|l|}{ Antithrombotic use $(n=296)$} \\
\hline - Warfarin alone & $36(12.2 \%)$ \\
\hline - Aspirin alone & $24(8.9 \%)$ \\
\hline - Warfarin + aspirin & $216(73.0 \%)$ \\
\hline - Dipyridamole & $13(4.4 \%)$ \\
\hline - Warfarin + aspirin+clopidogrel & $5(1.7 \%)$ \\
\hline - Other & $14(4.7 \%)$ \\
\hline - None & $10(3.4 \%)$ \\
\hline \multicolumn{2}{|l|}{ No. antithrombotic per patient $(n=296)$} \\
\hline . 0 & $9(3.0 \%)$ \\
\hline - 1 & $55(18.6 \%)$ \\
\hline .2 & $215(72.6 \%)$ \\
\hline .3 & $17(5.7 \%)$ \\
\hline $\begin{array}{l}\text { Mean no. antithrombotic agents per patient } \\
(n=296)\end{array}$ & 1.81 \\
\hline \multicolumn{2}{|l|}{ Was antithrombotic held pre-procedure? } \\
\hline - Held & $220(74.1 \%)$ \\
\hline - Continued & $30(10.8 \%)$ \\
\hline - Held \& bridged & $42(14.1 \%)$ \\
\hline - Unknown/not reported & $5(1.7 \%)$ \\
\hline \multicolumn{2}{|l|}{ Presenting symptoms of gastrointestinal bleeding ${ }^{1}$} \\
\hline - Symptomatic anemia & $150(50.1 \%)$ \\
\hline $\begin{array}{l}\text { - Without other overt signs of gastrointestinal } \\
\text { bleeding }\end{array}$ & $77(25.9 \%)$ \\
\hline - Melena & $142(47.8 \%)$ \\
\hline - Incidentally found anemia & $57(19.2 \%)$ \\
\hline - Hematochezia & $42(14.1 \%)$ \\
\hline - Hematemesis & $11(3.7 \%)$ \\
\hline - Coffee ground emesis & $4(1.3 \%)$ \\
\hline - Hypotension & $1(0.3 \%)$ \\
\hline $\begin{array}{l}\text { Total no. endoscopic procedures performed } \\
\text { (gastrointestinal bleeding events) }\end{array}$ & 533 \\
\hline \multicolumn{2}{|l|}{ Endoscopic procedures performed } \\
\hline - EGD & $228(42.8 \%)$ \\
\hline - Colonoscopy & $125(23.5 \%)$ \\
\hline - Push enteroscopy & $79(14.8 \%)$ \\
\hline - VCE & $68(12.8 \%)$ \\
\hline - Double-balloon enteroscopy & $33(6.2 \%)$ \\
\hline - Antegrade & $26(78.8 \%)$ \\
\hline - Retrograde & $7(21.2 \%)$ \\
\hline
\end{tabular}

- Table 5 (Continuation)

\section{Procedural characteristics ( $n=297$ gastrointestinal bleeding)}

$\%$ of procedures performed on inpatients

n (\%)

Top findings (all gastrointestinal bleeding procedures)

- GIAD

$121(42.2 \%)$

- Ulcer/erosions $38(13.2 \%)$

- Diffuse bleeding/no pinpoint source $26(9.1 \%)$

- GAVE

$17(5.9 \%)$

- Bleeding polyps $17(5.9 \%)$

- Overall diagnostic yield $49.5 \%$

Top locations of bleeding sources

- Stomach $113(39.4 \%)$

- Small bowel $83(28.9 \%)$

- Colon $50(17.4 \%)$

Total no. interventions (all gastrointestinal bleeding endoscopic procedures)

226

Top gastrointestinal bleeding interventions

- APC

- Endoclip $67(29.6 \%)$

- Bicap cautery $57(25.2 \%)$

Overall success rate $(178 / 185)$ $96.2 \%$

Overall adverse event rate, 30-day $35(6.6 \%)$

Non-procedure-related adverse event rate, 30-day

$20(3.8 \%)$

30-day adverse events (20/533 procedures)

- Death $14(2.6 \%)$

- CVA

- LVAD pump thrombosis

Procedural adverse events

$15(2.8 \%)$

Procedural adverse events (15/533 procedures)

- Bleeding $13(86.6 \%)$

- Perforation $1(6.6 \%)$

- Infection

$1(6.6 \%)$

EGD, Esophagogastroduodenoscopy; VCE, video capsule endoscopy; GIAD, gastrointestinal angiodysplasia; GAVE, gastric antral vascular ectasia; APC, Argon plasma coagulation; CVA, cerebrovascular accident.

Diagnostic yield for procedures was $49.5 \%$. (Note: procedures performed after source of bleeding found were not included in this calculation). Endoscopic interventions were $96.2 \%$ successful at hemostasis. (Note: there were several procedures with more than one endoscopic intervention; for this calculation each procedure was only once for the denominator). Procedurerelated (bleeding, infection, and perforation) adverse events were very minimal (2.8\%). Thirty-day post-procedure adverse events included LVAD pump thrombosis $(0.38 \%)$, CVA $(0.75 \%)$, and death $(2.6 \%)$. No reported deaths were associated with endoscopic procedures or interventions.

${ }^{1}$ Several patients had multiple presenting symptoms, therefore, the total will add up to more than $100 \%$. 
gastrointestinal bleeding in an LVAD patient, and as a result the authors advocate for assessment of gastrointestinal bleeding risk-factors when patients are being considered for LVAD placement. A nomogram (Utah Bleeding Risk Score) to predict risk of gastrointestinal bleeding in LVAD patients has been proposed in previous literature. This tool incorporates several predictive variables (e.g. coronary artery disease, history of bleeding events, age, etc.) that could potentially be used in the preimplantation period for risk-stratification [35]. Our data suggest that there may be a subset of LVAD patients that have a predisposition to develop a gastrointestinal bleeding. In this cohort, patients that experienced a bleeding event were more inclined to have recurrences; however, there was also a significant proportion (63.8\%) that never had a gastrointestinal bleeding develop in their post-implantation course. Independent risk factors of female sex, African-American race, DMII and pulmonary hypertension were each predictive of a gastrointestinal bleeding event. In existing literature, the only consistently reported risk factor of gastrointestinal bleeding in LVAD patients has been older age at time of implantation [6,16-19]. If the risk factors demonstrated in our cohort can be validated, appropriate counseling preimplantation and/or endoscopic screening for patients at high risk for post LVAD gastrointestinal bleeding may be considered; however, careful attention should be paid to the risk/benefit profile of such, as pre-LVAD implant patients are at high risk for cardiac events

There are several limitations of this study, inherent to its retrospective design. Our observations and clinical decision-making in management of gastrointestinal bleeding events reflect our single practice, thus results may be difficult to generalize to other institutions. Data were only collected on frequency of endoscopic procedures, not necessarily in the order in which they were performed.

\section{Conclusion}

As prevalence of heart failure in the general population continues to rise, the number of patients requiring LVAD implantation is also expected to increase. Evaluation and management of gastrointestinal bleeding in the LVADpopulation should be well understood by physicians practicing in high-volume LVAD institutions. Our study, with one of the largest cohorts (to our knowledge) of gastrointestinal bleeding in LVAD patients, demonstrated that endoscopy is a useful, effective, and most importantly safe modality, despite this being a high-risk population with necessity for long-term anticoagulant use. A systematic approach is necessary to manage the LVAD population, as risk of developing a gastrointestinal bleeding (most often recurrent GIAD) is inherent due to the requirement for longterm anticoagulation. Thus a multidisciplinary approach is key in management of these patients with close collaboration between the gastroenterology and cardiology teams regarding timing of endoscopy and anticoagulation management. Further studies need to be conducted regarding patient-specific factors that may predict a gastrointestinal bleeding event, the role of screening endoscopy, and the optimal standardized management approach for this population.

\section{Competing interests}

The authors declare that they have no conflict of interest.

References

[1] Mozaffarian D, Benjamin E], Go AS et al. Heart disease and stroke statistics-2016 update. A report from the American Heart Association. Circulation 2016; 133: e38-e360

[2] Heidenreich PA, Albert NM, Allen LA et al. Forecasting the impact of heart failure in the United States: a policy statement from the American Heart Association. Circ Heart Fail 2013; 6: 606-619

[3] Kirklin JK, Pagani FD, Kormos RL et al. Eighth annual INTERMACS report: Special focus on framing the impact of adverse events. J Heart Lung Transplant 2017; 36: 1080-1086

[4] Crow S, John R, Boyle A et al. Gastrointestinal bleeding rates in recipients of nonpulsatile and pulsatile left ventricular assist devices. J Thorac Cardiovasc Surg 2009; 137: 208-215

[5] Islam S, Cevik C, Madonna R et al. Left ventricular assist devices and gastrointestinal bleeding: a narrative review of case reports and case series. Clin Cardiol 2013; 36: 190-200

[6] Draper KV, Huang RJ, Gerson LB. Gl bleeding in patients with continuous-flow left ventricular assist devices: A systematic review and meta-analysis. Gastrointest Endosc 2014; 80: 435-446.e1

[7] Aggarwal A, Pant R, Kumar S et al. Incidence and management of gastrointestinal bleeding with continuous flow assist devices. Ann Thorac Surg 2012; 93: 1534-1540

[8] Morgan JA, Paone G, Nemeh HW et al. Gastrointestinal bleeding with the HeartMate II left ventricular assist device. J Heart Lung Transplant 2012; 31: 715-718

[9] Demirozu ZT, Radovancevic R, Hochman LF et al. Arteriovenous malformation and gastrointestinal bleeding in patients with the HeartMate II left ventricular assist device. J Heart Lung Transplant 2011; 30 : 849-853

[10] Goldstein DJ, Aaronson KD, Tatooles AJ et al. Gastrointestinal bleeding in recipients of the HeartWare Ventricular Assist System. JACC Heart Fail 2015; 3: 303-313

[11] Guha A, Eshelbrenner CL, Richards DM et al. Gastrointestinal Bleeding After Continuous-flow Left Ventricular Device Implantation: Review of Pathophysiology and Management. Methodist Debakey Cardiovasc J 2015; 11: 24-27

[12] Heyde EC. Gastrointestinal bleeding in aortic stenosis. N Engl J Med 1958; 259: 196

[13] Letsou GV, Shah N, Gregoric ID et al. Gastrointestinal bleeding from arteriovenous malformations in patients supported by the Jarvik 2000 axial-flow left ventricular assist device. J Heart Lung Transplant 2005; 24: 105-109

[14] Baldauf C, Schneppenheim R, Stacklies W et al. Shear-induced unfolding activates von Willebrand factor A2 domain for proteolysis. J Thromb Haemost 2009; 7: 2096-2105

[15] Uriel N, Pak SW, Jorde UP et al. Acquired von Willebrand syndrome after continuous-flow mechanical device support contributes to a high prevalence of bleeding during long-term support and at the time of transplantation. J Am Coll Cardiol 2010; 56: 1207-1213

[16] Shrode CW, Draper KV, Huang RJ et al. Significantly higher rates of gastrointestinal bleeding and thromboembolic events with left ventricular assist devices. Clin Gastroenterol Hepatol 2014; 12: 14611467

[17] Stern DR, Kazam J, Edwards P et al. Increased incidence of gastrointestinal bleeding following implantation of the HeartMate II LVAD. J Card Surg 2010; 25: 352-356 
[18] Kushnir VM, Sharma S, Ewald GA et al. Evaluation of GI bleeding after implantation of left ventricular assist device. Gastrointest Endosc 2012; 75: 973-979

[19] Demirozu ZT, Radovancevic R, Hochman LF et al. Arteriovenous malformation and gastrointestinal bleeding in patients with the HeartMate II left ventricular assist device. J Heart Lung Transplant 2011; 30: 849-853

[20] Aggarwal A, Pant R, Kumar S et al. Incidence and management of gastrointestinal bleeding with continuous flow assist devices. Ann Thorac Surg 2012; 93: 1534-1540

[21] Morgan JA, Panone G, Nemeh HW et al. Gastrointestinal bleeding with the HeartMate II left ventricular assist device. J Heart Lung Transplant 2012; 31: 715-718

[22] Sparrow CT, Nassif ME, Raymer DS et al. Pre-operative right ventricular dysfunction is associated with gastrointestinal bleeding in patients supported with continuous-flow left ventricular assist devices. JACC Heart Fail 2015; 3: 956-964

[23] Jabbar HR, Abbas A, Ahmed M et al. The incidence, predictors and outcomes of gastrointestinal bleeding in patients with left ventricular assist device (LVAD). Dig Dis Sci 2015; 60: 3697-3706

[24] Guha A, Eshelbrenner CL, Richards DM et al. Gastrointestinal bleeding after continuous-flow left ventricular assist device implantation: Review of pathophysiology and management. Methodist Debakey Cardiovasc J 2015; 11: 24-27

[25] Marsano J, Desai J, Chang S et al. Characteristics of gastrointestinal bleeding after placement of continuous-flow left ventricular assist device: A case series. Dig Dis Sci 2015; 60: 1859-1867

[26] Tarzia V, Dal Lin C, Bottio T et al. Occult gastrointestinal bleeding in patients with a left ventricular assist device axial flow pump: Diagnostic tools and therapeutic algorithm. J Thorac Cardiovasc Surg 2012; 143: e28-e31
[27] Elmunzer B], Padhya KT, Lewis J] et al. Endoscopic findings and clinica outcomes in ventricular assist device recipients with gastrointestinal bleeding. Dig Dis Sci 2011; 56: 3241-3246

[28] Axelrad JE, Pinsino A, Trinh PN et al. Limited usefulness of endoscopic evaluation in patients with continuous-flow left ventricular assist devices and gastrointestinal bleeding. J Heart Lung Transplant 2018; 37: 723-732

[29] Schulze PC, Kitada S, Clerkin K et al. Regional differences in recipient waitlist time and pre- and post- transplant mortality after the 2006 United Network for Organ Sharing policy changes in the donor heart allocation program. JACC Heart Fail 2014; 2: 166-177

[30] Kushnir VM, Sharma S, Ewald GA et al. Evaluation of GI Bleeding after implantation of left ventricular assist device. Gastrointest Endosc 2012: doi:10.1016/j.gie.2011.12.014

[31] Sarosiek K, Bogar L, Conn MI et al. An old problem with a new therapy: gastrointestinal bleeding in ventricular assist device patients and deep overtube-assisted enteroscopy. ASAIO J 2013; 59: 384-389

[32] Cushing K, Kushnir V. Gastrointestinal bleeding following LVAD placement from top to bottom. Dig Dis Sci 2016; 61: 1440-1447

[33] Guha A, Eshelbrenner CL, Richards DM et al. Gastrointestinal bleeding after continuous-flow left ventricular device implantation: Review of pathophysiology and management. Methodist Debakey Cardiovascular J 2015; 11: 24-27

[34] Thoban V, Shi Y, Rappelt M et al. The association of novel clinical factors and gastrointestinal bleeding among patients supported with continuous-flow left ventricular assist device therapy. J Card Surg 2019: doi:10.1111/jdocs.14062

[35] Yin MY, Ruckel S, Kfoury AG et al. Novel model to predict gastrointestinal bleeding during left ventricular device support. Circ Heart Fail 2018; 11: e005267 